\title{
A note on the number of long runs
}

\author{
S. NGuyen, X. Wang, and C. Martin
}

In this paper we present a simple derivation of the probability of attaining a run of $r$ heads when tossing a coin $n$ times. We use this result to establish other properties of coin tossing. Using the same techniques we find the probability of runs of either heads or tails. We give a complete analysis of both recurrences. In addition we construct the generating functions for the recurrences.

\section{Introduction}

It is well known that if a coin is tossed many times there is a reasonable probability of long consecutive runs. Feller [8] studied this problem as has many other mathematicians and physicists. Feller was mostly concerned with the recurrent nature of the phenomena and only visited the probabilities through the generating functions. However the problem of calculating the probabilities has a reputation of being hard and often the probabilities are calculated using Monte Carlo simulations, [1], [5], [7] and [9]. In this paper we establish a simple recursion that calculates the probabilities and from this we calculate the exact solution of the probabilities as a function of $r$ and $n$.

One can think of the recurrences as being high order difference equations and so we reduce them to first order systems and use system theoretic techniques to analyze the asymptotic behavior. Here the largest eigenvalue plays a major role in the behavior. We also construct the generating functions. Generating functions are not usually considered in system theory but since they are the major part of Feller's analysis we calculate them here. We show that the radius of convergence of the McLaurin series is given by the reciprocal of the largest eigenvalue of the characteristic function.

\section{The basic recursion for runs of heads}

In this section we establish the basic recurrence. The key to the construction is to go carefully from sequences of length $n$ to sequences of length $n+1$. We first establish the recurrence for runs of heads and then we construct 
the recurrences for long runs of either heads or tails. The recurrences are calculated for the probabilities of a run of $r$ heads and for the case of runs of either heads or tails. For convenience we will work with 0's and 1's rather than heads and tails.

Definition 2.1. let $N(n, r)$ be the number of sequences of length $n$ of 0 's and 1's that have at least one consecutive subsequence of 1's of length greater than or equal to $r$.

We will prove the following theorem.

Theorem 2.2. The following recursion holds when $n \geq r$

$$
N(n+1, r)=2 N(n, r)-N(n-r, r)+2^{n-r} .
$$

When $n \leq r$ the following equalities hold:

$$
N(0, r)=N(1, r)=\cdots=N(r-1, r)=0
$$

and $N(r, r)=1$.

Proof. The equalities are clear. There cannot be a sequence of 1's of length $r$ if the total length is less than $r$. Now consider the set of all sequences of length $n, S_{n}$. Clearly $S_{n+1}$ is a disjoint union of $A(0, n)$ and $A(1, n)$, where

$$
A(0, n)=\left\{(0, \alpha): \alpha \in S_{n}\right\} \quad \text { and } \quad A(1, n)=\left\{(1, \alpha): \alpha \in S_{n}\right\} .
$$

By adding a 0 we have not created or destroyed any sequence $\alpha$ with a run of 1's of length greater than or equal to $r$. Thus $A(0, n)$ contains exactly $N(n, r)$ such sequences. By adding a 1 we have not destroyed any sequences containing 1's of length $r$ or greater. So $A(1, n)$ contains at least $N(n, r)$ desired sequences. The only way we can create additional sequences is if the beginning subsequence of $\alpha$ is $1,1, \ldots, 1,0$ with $r-1$ ones. The 0 is necessary for if not then it was already of length $r$. Now the remainder of the sequence is of length $n-r$ and there are $2^{n-r}$ such sequences. Among them, those containing 1's of length $r$ or greater are already counted. Thus the set $A(1, n)$ contains $N(n, r)+2^{n-r}-N(n-r, r)$ sequences with runs of length $r$ or greater. The theorem follows. 


\subsection{The recurrence for the probabilities}

Let $P(n, r)$ be the probability that a sequence of length $\mathrm{n}$ contains a run of length $r$ or greater. Using the recurrence (2.1) and dividing both sides by $2^{n+1}$ we have the following theorem.

Theorem 2.3. When $n \geq r$ the following recurrence holds

$$
P(n+1, r)=P(n, r)+2^{-r-1}(1-P(n-r, r))
$$

whenever $n \geq r$. When $n \leq r$ the following equalities hold:

$$
P(0, r)=P(1, r)=\cdots=P(r-1, r)=0
$$

and $P(r, r)=2^{-r}$.

Proof. We make the following calculation.

$$
\begin{aligned}
P(n+1, r)=\frac{N(n+1, r)}{2^{n+1}} & =\frac{2 N(n, r)}{2^{n+1}}+\frac{2^{n-r}}{2^{n+1}}-\frac{N(n-r, r)}{2^{n+1}} \\
& =P(n, r)+\frac{1}{2^{r+1}}-\frac{N(n-r, r)}{2^{n-r+r+1}} \\
& =P(n, r)+\frac{1}{2^{r+1}}-\frac{P(n-r, r)}{2^{r+1}} \\
& =P(n, r)+\frac{1}{2^{r+1}}(1-P(n-r, r))
\end{aligned}
$$

\subsection{Heads or tails}

We now turn to the probability of a run of length $r$ of heads or tails when a coin is tossed $n$ times. Let

$$
H(n, r)
$$

be the number of sequences of length $n$ that contain at least one run of length at least $r$. We will prove the following theorem which is similar to Theorem 2.2. 
Theorem 2.4. The following recursion holds

$$
H(n+1, r)=2 H(n, r)-H(n-r+1, r)+2^{n-r+1}
$$

whenever $n \geq r$. The following equalities hold: $H(0, r)=H(1, r)=\cdots=$ $H(r-1, r)=0$ and $H(r, r)=2$.

Proof. As before we split the set of sequences of length $n+1$ in to two parts, $A(0, n)$ and $A(1, n)$. Now each $A(x, n)$ contains $H(n, r)$ sequences with a run of 0 's or 1 's of length $r$. The only way that adding an initial 0 to a sequence $\alpha$ can add a sequence with a run of length $r$ is if the $\alpha$ is of the form

$$
\left(0, \ldots, 0,1, x_{1}, \ldots, x_{n-r}\right)
$$

with $r-1$ initial 0's, and there are $2^{n-r}$ many such sequences. Similarly, the only new one in $A(1, n)$ are those with $\alpha=\left(1, \ldots, 1,0, x_{1}, \ldots, x_{n-r}\right)$ and $r-1$ initial 1's. Together there are $2^{n-r+1}$ many such sequences. Among them, those $\left(1, x_{1}, \ldots, x_{n-r}\right)$ or $\left(0, x_{1}, \ldots, x_{n-r}\right)$ already containing run of length at least $r$ are already counted, and there are $H(n-r+1, r)$ many such sequences. The theorem follows.

\subsection{The recurrence for the probabilities of runs of heads or tails}

We will prove the following theorem which is the analog of Theorem 2.3. Let $P H(n, r)$ be the probability of a sequence of length $\mathrm{n}$ having a run of zeros or ones of length $\mathrm{r}$ or greater.

Theorem 2.5. The recurrence for the probability of a sequence of 0 's and 1 's having runs of 0 's or 1 's of length $r$ or greater is given by

$$
P H(n+1, r)=P H(n, r)+2^{-r}(1-P H(n-r+1, r))
$$

with initial values

$$
P H(0, r)=\cdots=P H(r-1, r)=0, \quad P H(r, r)=2^{-r+1} .
$$

Proof. The proof is the same as before.

\section{The difference equations}

In this secton we give a very complete analysis of the location of the eigenvalues of the systems. We thus gain a very precise understanding of the 
speed of convergence. The analysis is based on, among other tools, root locus techniques. For reasons of readability the proof of the major theorems are moved to the appendix.

The recurrences may be thought of as higher order difference equations and as is now standard in systems theory we rewrite them as a system of first order equations. We write the recurrence (2.1) as

$$
x_{n+1}=2 x_{n}-x_{n-r}+2^{n-r}
$$

and convert it to a first order equation

(3.1)

$$
\left(\begin{array}{c}
x_{n-r+1} \\
x_{n-r+2} \\
\vdots \\
\vdots \\
x_{n} \\
x_{n+1}
\end{array}\right)=\left(\begin{array}{ccccc}
0 & 1 & 0 & \cdots & 0 \\
0 & 0 & 1 & \cdots & 0 \\
\vdots & \vdots & \ddots & \ddots & \vdots \\
\vdots & \vdots & & \ddots & 1 \\
-1 & 0 & 0 & \cdots & 2
\end{array}\right)\left(\begin{array}{c}
x_{n-r} \\
x_{n-r+1} \\
\vdots \\
\vdots \\
x_{n-1} \\
x_{n}
\end{array}\right)+\left(\begin{array}{c}
0 \\
0 \\
\vdots \\
\vdots \\
0 \\
1
\end{array}\right) 2^{n-r}
$$

with initial data

$$
\left(x_{0}, x_{1}, \ldots, x_{r-1}, x_{r}\right)^{T}=(0,0, \ldots, 0,1)^{T}
$$

The characteristic equation is given by

$$
\operatorname{det}\left(\begin{array}{ccccc}
-\lambda & 1 & 0 & \cdots & 0 \\
0 & -\lambda & 1 & \cdots & 0 \\
\vdots & \vdots & \ddots & \ddots & \vdots \\
\vdots & \vdots & & -\lambda & 1 \\
-1 & 0 & 0 & \cdots & 2-\lambda
\end{array}\right)=0
$$

and so the characteristic equation is

$$
\lambda^{r+1}-2 \lambda^{r}+1=0
$$

Let $p(t)=t^{r+1}-2 t^{r}+1$ and note that $p(1)=0$ and if $t>1 p(t)>0$. Now the extremals occur at

$$
t_{0}=\frac{2 r}{r+1}>1
$$

for $r>2$. Note that $p\left(t_{0}\right)<0$ for $r>2$ and hence there are zeros outside the unit circle and the system is unstable. We will prove the following theorem. 
Theorem 3.1. For every $r \geq 2$ the zeros of the polynomial $p(t)=t^{r+1}-$ $2 t^{r}+1$ lie in the circle centered at 0 with radius 2 . The largest real root lies in the open interval $\left(\frac{2 r}{r+1}, 2\right)$.

Key to the proof is the following theorem due to M. Dehmer, [2] and [3]. The proof of the theorem is found in the references.

Theorem 3.2 (Dehmer). Let

$$
f(z)=a_{n} z^{n}+a_{n-1} z^{n-1}+\cdots+a_{1} z+a_{0}, \quad a_{n} a_{n-1} \neq 0
$$

be a complex polynomial. All zeros of $f(z)$ lie in the closed disk

$$
K\left(0, \max \left(1, \delta_{2}\right)\right)
$$

where $\delta_{2}$ (besides $\delta_{1}=1$ ) is a positive root of the equation

$$
z^{n+1}-\left(1+M_{2}\right) z^{n}+M_{2}=0 \quad \text { and } \quad M_{2}=\max _{0 \leq j \leq n-1}\left|\frac{a_{j}}{a_{n}}\right| .
$$

The bound is sharp for all polynomials of the form

$$
f(z)=a z^{n}-b\left(z^{n-1}+z^{n-2}+\cdots+1\right), \quad a, b \neq 0 .
$$

We begin the proof of the theoroem by proving the following lemma.

Lemma 3.3. The largest real zero of $p(z)=z^{r+1}-2 z^{r}+1$ lies in the interval $\left(\frac{2 r}{r+1}, 2\right)$.

Proof. We note that $p(0)=p(2)=1>0$ and that $p(1)=0$. the critical values are $t=0$ (with multiplicity $r-1$ ) and $t_{0}=\frac{2 r}{r+1}$. Evaluating $p\left(\frac{2 r}{r+1}\right)$ we see after some calculation that it is negative for $r \geq 2$. Thus, there is a zero in the interval $\left(\frac{2 r}{r+1}, 2\right)$. So $p$ is a minimum at this point and there can be no other zeros in the interval. The lemma follows.

Proof of theorem. We note that

$$
t^{r+1}-2 t^{r}+1=(t-1)\left(t^{r}-\left(t^{r-1}+t^{r-2}+\cdots+1\right)\right) .
$$

Applying Dehmer's theorem to $t^{r}-\left(t^{r-1}+t^{r-2}+\cdots+1\right)$ the theorem follows.

By using root locus technique [6] we have a tighter estimate of the locations of the zeros of $p(t)$. 
Theorem 3.4. For $r>2$, the matrix

$$
A=\left(\begin{array}{ccccc}
0 & 1 & 0 & \ldots & 0 \\
0 & 0 & 1 & \ldots & 0 \\
\vdots & \vdots & \ddots & \ddots & \vdots \\
\vdots & \vdots & & \ddots & 1 \\
-1 & 0 & 0 & \ldots & 2
\end{array}\right)
$$

has $r+1$ simple eigenvalues. It has 2 positive eigenvalues

$$
\lambda_{1}=1 \quad \text { and } \quad \lambda_{2} \in\left(2-\frac{\left(1+\frac{1}{r}\right)^{r}}{2^{r}}, 2-\frac{1}{2^{r}}\right)
$$

and it has a third negative real eigenvalue if $r$ is even. All the other eigenvalues are complex. Except for $\lambda_{1}$ and $\lambda_{2}$, all the other eigenvalues are located inside the unit circle $|\lambda|=1$ of the complex plane. For each eigenvalue $\lambda_{j}$, the associated eigenvector is given by

$$
\xi_{j}=\left(1, \lambda_{j}, \lambda_{j}^{2}, \ldots, \lambda_{j}^{r}\right)^{T}
$$

The proof is in the appendix.

Now consider the system (3.1) and its formal solution.

Theorem 3.5. Let $\lambda_{j}, j=1, \ldots, r+1$ be the eigenvalues of the $(r+1) \times$ $(r+1)$ matrix $A$ in (3.3). Then $N(n, r)$ is given by

$$
N(n, r)=2^{n-r} \sum_{j=1}^{r+1} \frac{\lambda_{j}\left(1+\left(\lambda_{j} / 2\right)^{n-r}-2\left(\lambda_{j} / 2\right)^{n-r+1}\right)}{\left(2-\lambda_{j}\right)\left((r+1) \lambda_{j}-2 r\right)}
$$

The proof is in the appendix.

For the case of heads or tails, the recurrence is given by

$$
x_{n+1}=2 x_{n}-x_{n-r+1}+2^{n-r+1} \text {. }
$$


So the associated first order system is (3.6)

$$
\left(\begin{array}{c}
x_{n-r+2} \\
x_{n-r+3} \\
\vdots \\
\vdots \\
x_{n} \\
x_{n+1}
\end{array}\right)=\left(\begin{array}{ccccc}
0 & 1 & 0 & \cdots & 0 \\
0 & 0 & 1 & \cdots & 0 \\
\vdots & \vdots & \ddots & \ddots & \vdots \\
\vdots & \vdots & & \ddots & 1 \\
-1 & 0 & 0 & \cdots & 2
\end{array}\right)\left(\begin{array}{c}
x_{n-r+1} \\
x_{n-r+2} \\
\vdots \\
\vdots \\
x_{n-1} \\
x_{n}
\end{array}\right)+\left(\begin{array}{c}
0 \\
0 \\
\vdots \\
\vdots \\
0 \\
1
\end{array}\right) 2^{n-r+1}
$$

with initial data

$$
\left(x_{1}, \ldots, x_{r-1}, x_{r}\right)^{\prime}=(0,0, \ldots, 0,2)^{\prime} .
$$

Theorem 3.6. Let $\lambda_{j}, j=1, \ldots, r$ be the eigenvalues of the $r \times r$ matrix A in (3.3). Then

$$
H(n, r)=2^{n-r+1} \sum_{j=1}^{r} \frac{\lambda_{j}\left(1+\left(\lambda_{j} / 2\right)^{n-r}-2\left(\lambda_{j} / 2\right)^{n-r+1}\right)}{\left(2-\lambda_{j}\right)\left(r \lambda_{j}-2(r-1)\right)}
$$

The proof is in the appendix.

\section{Corollary 3.7.}

$$
P(n, r)=\frac{1}{2^{r}} \sum_{j=1}^{r+1} \frac{\lambda_{j}\left(1+\left(\lambda_{j} / 2\right)^{n-r}-2\left(\lambda_{j} / 2\right)^{n-r+1}\right)}{\left(2-\lambda_{j}\right)\left((r+1) \lambda_{j}-2 r\right)}
$$

where $\lambda_{1}, \ldots, \lambda_{r+1}$ are eigenvalues of the $(r+1) \times(r+1)$ matrix (3.3).

$$
P H(n, r)=\frac{1}{2^{r-1}} \sum_{j=1}^{r} \frac{\mu_{j}\left(1+\left(\mu_{j} / 2\right)^{n-r}-2\left(\mu_{j} / 2\right)^{n-r+1}\right)}{\left(2-\mu_{j}\right)\left(r \mu_{j}-2(r-1)\right)}
$$

where $\mu_{1}, \ldots, \mu_{r}$ are eigenvalues of the $r \times r$ matrix (3.3).

\subsection{The difference equation for the probabilities}

Using standard notation for equation (2.2) we have

$$
z_{n+1}=z_{n}+2^{-r-1}\left(1-z_{n-r}\right)
$$


Checking for equilibria we set

$$
x=x+2^{-r-1}(1-x)
$$

and from this we see that the only equilibrium is at $x=1$. Expanding around $x=1$ we have the equation

$$
\left(z_{n+1}-1\right)=\left(z_{n}-1\right)+2^{-r-1}\left(1-z_{n-r}\right)
$$

and letting

$$
y_{n}=z_{n}-1
$$

we have the new equation

$$
y_{n+1}=y_{n}-2^{-r-1} y_{n-r} .
$$

Writing this as a first order equation we have

$(3.11) \quad\left(\begin{array}{c}y_{n-r+1} \\ y_{n-r+2} \\ \vdots \\ y_{n} \\ y_{n+1}\end{array}\right)=\left(\begin{array}{ccccc}0 & 1 & 0 & \cdots & 0 \\ 0 & 0 & 1 & \cdots & 0 \\ \vdots & \vdots & \ddots & \ddots & \vdots \\ \vdots & \vdots & & 0 & 1 \\ -2^{-r-1} & 0 & \cdots & 0 & 1\end{array}\right)\left(\begin{array}{c}y_{n-r} \\ y_{n-r+1} \\ \\ \vdots \\ y_{n-1} \\ y_{n}\end{array}\right)$

with initial data

$$
\left(y_{0}, y_{1}, \ldots, y_{r-1}, y_{r}\right)^{\prime}=(-1,-1, \ldots,-1,0)^{\prime}
$$

The characteristic is given by

$$
0=\left|\begin{array}{ccccc}
-\lambda & 1 & 0 & \cdots & 0 \\
0 & -\lambda & 1 & \cdots & 0 \\
\vdots & \vdots & \ddots & \ddots & \vdots \\
\vdots & \vdots & & -\lambda & 1 \\
-2^{-r-1} & 0 & \cdots & 0 & 1-\lambda
\end{array}\right|
$$




$$
\begin{aligned}
& =(-1)^{r+1} 2^{-r-1}\left|\begin{array}{cccc}
1 & 0 & \ldots & 0 \\
-\lambda & 1 & \ldots & 0 \\
\vdots & \ddots & \ddots & \vdots \\
0 & \cdots & -\lambda & 1
\end{array}\right|+(1-\lambda)\left|\begin{array}{cccc}
-\lambda & 1 & \cdots & 0 \\
0 & -\lambda & \ddots & \vdots \\
\vdots & \vdots & \ddots & 1 \\
0 & 0 & \cdots & -\lambda
\end{array}\right| \\
& =(-1)^{r+1} 2^{-r-1}+(-1)^{r}(1-\lambda) \lambda^{r} \\
& =(-1)^{r+1}\left(\lambda^{r+1}-\lambda^{r}+2^{-r-1}\right)
\end{aligned}
$$

Now let

$$
p(t)=t^{r+1}-t^{r}+2^{-r-1} .
$$

The extremal value occurs at

$$
t_{0}=\frac{r}{r+1}
$$

and

$$
p\left(t_{0}\right)=\left(\frac{r}{r+1}\right)^{r}\left(\frac{-1}{r+1}\right)+2^{-r-1} .
$$

This is negative for $r$ greater than 1 so there exists two zeros in the interval $[0,1]$ because $p(0)=p(1)=2^{-r-1}>0$. Now if $r$ is even the $p(-1)=-1-$ $1+2^{-r-1}<0$ and there is a zero in the interval $[-1,0]$ and there are no real zeros outside the interval $(-1,1)$. If $r$ is odd then also there are no real zeros outside the unit interval.

Theorem 3.8. For $r>1, p(t)$ defined in (3.12) has 2 positive zeros

$$
t_{1} \in\left(1-\frac{\left(1+\frac{1}{r}\right)^{r}}{2^{r+1}}, 1-\frac{1}{2^{r+1}}\right) \quad \text { and } \quad t_{2} \in\left(\frac{1}{2^{1+\frac{1}{r}}}, \frac{1}{2^{1+\frac{1}{r}}}(1+r)^{1 / r}\right) .
$$

If $r$ is even, $p(t)$ also has a negative real zero

$$
t_{3} \in\left(\frac{-1}{2^{1+\frac{1}{r}}}, \frac{-1}{2^{1+\frac{2}{r}}}\right)
$$

All other zeros are complex and located inside of the circle

$$
|t|=\frac{(1+r)^{1 / r}}{2^{1+\frac{1}{r}}}
$$

The proof is in the appendix. 
Since all the eigenvalues of the matrix in the system (3.11) are insider the unit circle, the system is stable and

$$
\lim _{n \rightarrow \infty}\left(y_{n-r}, \ldots, y_{n}\right)^{T}=0 .
$$

Therefore

\section{Corollary 3.9.}

$$
\lim _{n \rightarrow \infty} P(n, r)=\lim _{n \rightarrow \infty} P H(n, r)=1 .
$$

Proof. Note that $P(n, r)=1+y_{n}$ and $P H(n, r) \geq P(n, r)$.

\section{The generating function}

Generating functions have long been a standard tool in probability but have not been extensively used in systems theory. Here we will generate them using the recurrences and will compare them to those in Feller. We begin by letting

$$
\begin{aligned}
F_{r}(t) & =\sum_{n=1}^{\infty} N(n, r) t^{n} \\
& =\sum_{n=1}^{r} N(n, r) t^{n}+\sum_{n=r+1}^{\infty} N(n, r) t^{n} \\
& =t^{r}+\sum_{n=r+1}^{\infty} N(n, r) t^{n}
\end{aligned}
$$

Note that

$$
F_{r}\left(\frac{t}{2}\right)=\sum_{n=1}^{\infty} P(n, r) t^{m}=G_{r}(t)
$$

so that the generating function for the probabilities is constructed directly from the recurrence for the number of sequences. We will find the generating functions associated with

$$
N(n+1, r)=2 N(n, r)-N(n-r, r)+2^{n-r} .
$$


We now construct the multiples of $F$ that we need for the recurrence.

$$
\begin{aligned}
t^{-1} F(t) & =\sum_{n=r}^{\infty} N(n, r) t^{n-1} \quad(\text { let } m=n-1) \\
& =\sum_{m=r-1}^{\infty} N(m+1, r) t^{m} . \\
t^{r} F(t) & =\sum_{n=0}^{\infty} N(n, r) t^{n+r} \quad(\text { let } m=n-r) \\
& =\sum_{m=r}^{\infty} N(m-r, r) t^{m} .
\end{aligned}
$$

Now let $h(t)=(1-2 t)^{-1}$.

$$
\begin{aligned}
t^{r} h(t) & =\sum_{n=0}^{\infty} 2^{n} t^{n+r} \quad(\text { let } m=n+r) \\
& =\sum_{m=r}^{\infty} 2^{m-r} t^{m} .
\end{aligned}
$$

We now construct the generating function. Combine (4.1),(4.2),(4.3)

$$
\begin{aligned}
& t^{-1} F(t)-2 F(t)+t^{r} F(t)-t^{r} h(t) \\
= & N(r-1+1, r) t^{r-1} \\
& +\sum_{m=r}^{\infty}\left(N(m+1, r)-2 N(m, r)+N(m-r, r)-2^{m-r}\right) t^{m} \\
= & t^{r-1}
\end{aligned}
$$

Multiplying by $t$ we have

$$
F(t)\left(1-2 t+t^{r+1}\right)=t^{r+1} h(t)+t^{r}
$$

We have then

$$
\begin{aligned}
F(t) & =\frac{t^{r+1}+(1-2 t) t^{r}}{(1-2 t)\left(1-2 t+t^{r+1}\right)} \\
& =\frac{t^{r}(1-t)}{(1-2 t)\left(1-2 t+t^{r+1}\right)}
\end{aligned}
$$


Now let $p(t)=1-2 t+t^{r+1}$ and calculate

$$
p\left(x^{-1}\right)=1-2 x^{-1}+x^{-r-1}=x^{-r-1}\left(x^{r+1}-2 x^{r}+1\right)
$$

and recall that $x^{r+1}-2 x^{r}+1$ is the characteristic polynomial of the recursion and the associated first order linear system. Thus the radius of the Maclurian systems is the minimum of $1 / 2$ and the reciprocal of the largest eigenvalue of the linear system.

Since $F\left(\frac{t}{2}\right)=\sum_{n=0}^{\infty} P(n, r) t^{n}$ we have the generating function for the probability of a run of $r$ heads in a sequence of $n$ trials is given by

$$
G(t)=\frac{t^{r}(2-t)}{(1-t)\left(2^{r+1}-2^{r+1} t+t^{r+1}\right)}
$$

We now turn to calculating the generating function for the number of runs of length $r$ of heads or tails in a sequence of length of $n$. Recall that the recurrence is given by

$$
\begin{aligned}
H(n+1, r)-2 H(n, r)+H(n-r+1, r)-2^{n-r+1}=0 . & \\
J(t) & =\sum_{m=1}^{\infty} H(m, r) t^{m}=\cdots=\sum_{m=r}^{\infty} H(m, r) t^{m} \\
& =\sum_{m=r+1}^{\infty} H(m, r) t^{m}+2 t^{r} \\
t^{-1} J(t) & =\sum_{m=r-1}^{\infty} H(m+1, r) t^{m}=2 t^{r-1}+\sum_{m=r}^{\infty} H(m+1, r) t^{m} \\
t^{r-1} J(t) & =\sum_{m=r}^{\infty} H(m-r+1) t^{m} \\
t^{r-1} h(t) & =\sum_{m=r-1}^{\infty} 2^{m-r+1} t^{m}=t^{r-1}+\sum_{m=r}^{\infty} 2^{m-r+1} t^{m}
\end{aligned}
$$

Thus using the above we have

$$
t^{-1} J(t)-2 J(t)+t^{r-1} J(t)-t^{r-1} h(t)=2 t^{r-1}-t^{r-1}=t^{r-1}
$$

and multiplying both sides by $t$ we have

$$
J(t)-2 t J(t)+t^{r} J(t)-t^{r} h(t)=t^{r}
$$


So

$$
J(t)=\frac{t^{r}(1+h(t))}{1-2 t+t^{r}}=\frac{2 t^{r}(1-t)}{(1-2 t)\left(1-2 t+t^{r}\right)} .
$$

\section{Numerical results}

In this chapter we present 4 tables. The two larger tables are simple tables giving the probability of a run of $r$ heads or a run of $r$ heads or tails occurring when a coin is tossed $n$ times. The two short tables show how large $n$ must be for a run of length $r$ to have a probability of .5 of occurring. The tables were produced using Excel and the numbers were given to 10 decimal pales. Those numbers were truncated to 3 decimal places for reasons of space. Noted that they were not rounded. Tables 2 and 4 are just subtables of Tables 1 and 3 .

\begin{tabular}{|c||c|c|c|c|c|c|c|c|}
\hline $\mathrm{n} / \mathrm{r}$ & 2 & 3 & 4 & 5 & 6 & 7 & 8 & 9 \\
\hline \hline 2 & 0.25 & 0 & 0 & 0 & 0 & 0 & 0 & 0 \\
3 & 0.375 & 0.125 & 0 & 0 & 0 & 0 & 0 & 0 \\
4 & 0.5 & 0.187 & 0.062 & 0 & 0 & 0 & 0 & 0 \\
5 & 0.593 & 0.25 & 0.093 & 0.0312 & 0 & 0 & 0 & 0 \\
6 & 0.671 & 0.312 & 0.125 & 0.0468 & 0.0156 & 0 & 0 & 0 \\
7 & 0.734 & 0.3671 & 0.156 & 0.062 & 0.0234 & 0.007 & 0 & 0 \\
8 & 0.785 & 0.417 & 0.185 & 0.078 & 0.031 & 0.011 & 0.003 & 0 \\
9 & 0.826 & 0.464 & 0.213 & 0.093 & 0.039 & 0.015 & 0.005 & 0.001 \\
10 & 0.859 & 0.507 & 0.241 & 0.108 & 0.046 & 0.019 & 0.007 & 0.002 \\
22 & 0.988 & 0.819 & 0.503 & 0.272 & 0.135 & 0.065 & 0.031 & 0.014 \\
45 & 0.999 & 0.973 & 0.779 & 0.506 & 0.283 & 0.147 & 0.074 & 0.036 \\
89 & 0.999 & 0.999 & 0.953 & 0.764 & 0.500 & 0.285 & 0.151 & 0.077 \\
178 & 1 & 0.999 & 0.998 & 0.947 & 0.758 & 0.500 & 0.288 & 0.154 \\
356 & 1 & 1 & 0.999 & 0.997 & 0.943 & 0.755 & 0.500 & 0.290 \\
711 & 1 & 1 & 1 & 0.999 & 0.996 & 0.941 & 0.752 & 0.500 \\
\hline
\end{tabular}

Table 1: Probability of a run of heads of length $r$ when a coin is tossed $n$ times.

Let

$$
N(r, .5)=\min \{P(n, r): P(n, r) \geq .5\}
$$

Note that in Table 2 and Table 4 the numbers double at each step after some initial adjustment. We are led to the the following conjecture. 


\begin{tabular}{|c||c|c|c|c|c|c|c|c|}
\hline $\mathrm{r}$ & 2 & 3 & 4 & 5 & 6 & 7 & 8 & 9 \\
\hline $\mathrm{N}(\mathrm{r}, .5)$ & 4 & 10 & 22 & 45 & 89 & 178 & 356 & 711 \\
\hline
\end{tabular}

Table 2: For runs of heads of length $\mathrm{r}$ the smallest $\mathrm{n}$ for which the probability is greater than or equal .5.

\begin{tabular}{|c||c|c|c|c|c|c|c|c|}
\hline $\mathrm{n} / \mathrm{r}$ & 2 & 3 & 4 & 5 & 6 & 7 & 8 & 9 \\
\hline \hline 2 & 0.5 & 0 & 0 & 0 & 0 & 0 & 0 & 0 \\
3 & 0.75 & 0.25 & 0 & 0 & 0 & 0 & 0 & 0 \\
4 & 0.875 & 0.375 & 0.125 & 0 & 0 & 0 & 0 & 0 \\
5 & 0.937 & 0.5 & 0.187 & 0.062 & 0 & 0 & 0 & 0 \\
6 & 0.968 & 0.593 & 0.25 & 0.093 & 0.031 & 0 & 0 & 0 \\
7 & 0.984 & 0.671 & 0.312 & 0.125 & 0.046 & 0.015 & 0 & 0 \\
8 & .992 & 0.734 & 0.367 & 0.156 & 0.062 & 0.023 & 0.007 & 0 \\
9 & 0.996 & 0.785 & 0.417 & 0.187 & 0.078 & 0.031 & 0.011 & 0.003 \\
10 & 0.998 & 0.826 & 0.464 & 0.216 & 0.093 & 0.039 & 0.015 & 0.005 \\
11 & 0.999 & 0.859 & 0.507 & 0.245 & 0.109 & 0.046 & 0.019 & 0.007 \\
23 & 0.999 & 0.988 & 0.819 & 0.515 & 0.275 & 0.136 & 0.065 & 0.031 \\
45 & 1 & 0.999 & 0.971 & 0.784 & 0.503 & 0.279 & 0.144 & 0.072 \\
90 & 1 & 0.999 & 0.999 & 0.959 & 0.770 & 0.503 & 0.286 & 0.151 \\
179 & 1 & 1 & 0.999 & 0.998 & 0.950 & 0.761 & 0.501 & 0.289 \\
357 & 1 & 1 & 1 & 0.999 & 0.997 & 0.944 & 0.756 & 0.500 \\
\hline \hline
\end{tabular}

Table 3: Probability of a run of heads or tails of length $r$ when a coin is tossed $n$ times.

\begin{tabular}{|c||c|c|c|c|c|c|c|c|}
\hline $\mathrm{r}$ & 2 & 3 & 4 & 5 & 6 & 7 & 8 & 9 \\
\hline $\mathrm{N}(\mathrm{r}, .5)$ & 2 & 5 & 11 & 23 & 45 & 90 & 179 & 357 \\
\hline
\end{tabular}

Table 4: For runs of heads of length $\mathrm{r}$ the smallest $\mathrm{n}$ for which the probability is greater than or equal .5.

\section{Conjecture 5.1.}

$$
\lim _{r \rightarrow \infty} \frac{N(r+1, .5)}{N(r, .5)}=2
$$




\section{Conclusion}

In this paper we have presented simple recursions for the probability of long runs of successes or failures in a Bernoulli trial. The main contribution of the paper are the recursions and the analysis of the recursions. The roots of the characteristic are characterized and their influence on convergence is shown. Since the recurrences are linear the first order equivalent systems are in the form of forced linear systems and through this there is a simple connection to linear control theory. The generating functions for the recurances are calculated. Finally in calculating the tables it was noticed that there seemed to be a simple relationship between the number of coin tosses needed to have probability of .5 of a run of successes. We do not know how to prove the conjecture and would be very interested in a proof of the conjecture.

\section{Appendix: Proofs of theorems}

Proof of Theorem 3.4. The equation $p(t)=0$ can be written as $t^{r}(t-2)=$ -1 . We use root locus technique [6] to analyze the zeros of $t^{r}(t-2)=k$. When $k=0$, the zeros are 2 and 0 with multiplicity $r$. For negative $k$, part of real axis is on the root locus if and only if it is to the left of odd number of zeros of $t^{r}(t-2)$. So when $k$ changes from 0 to -1 , the zero at 2 moves toward the left, one of the multiple zeros at 0 breaks way and moves toward the right to 1 , and when $r$ is even, another of the multiple zeros at 0 breaks way and moves toward the left. All the other multiple zeros at 0 break away and become complex. Therefore all the eigenvalues of $A$ are simple.

The equation $p(t)=0$ can be written as

$$
t=2-\frac{1}{t^{r}}
$$

Consider the sequence defined by

$$
x_{n+1}=2-\frac{1}{x_{n}^{r}} .
$$

Note that $p^{\prime}(t)>0$ for $t>t_{0}=\frac{2 r}{r+1}$. So $p(t)$ is increasing, and

$$
p(t)<0 \text { for } t \in\left[t_{0}, \lambda_{2}\right) \text { and } p(t)>0 \text { for } t \in\left(\lambda_{2}, \infty\right) \text {. }
$$

Since

$$
x_{n+1}-x_{n}=2-\frac{1}{x_{n}^{r}}-x_{n}=\frac{-p\left(x_{n}\right)}{x_{n}^{r}},
$$


$x_{n+1}>x_{n}$ if $x_{n} \in\left[t_{0}, \lambda_{2}\right)$, and $x_{n+1}<x_{n}$ if $x_{n} \in\left(\lambda_{2}, \infty\right)$. Since

$$
x_{n+1}-x_{n}=\frac{1}{x_{n-1}^{r}}-\frac{1}{x_{n}^{r}}=\left(x_{n}-x_{n-1}\right) \frac{x_{n}^{r-1}+x_{n}^{r-2} x_{n-1}+\cdots+x_{n-1}^{r-1}}{x_{n-1}^{r} x_{n}^{r}},
$$

$\left\{x_{n}\right\}$ is a monotone sequence for any initial $x_{0} \in\left[t_{0}, \infty\right)$. By (7.2) and (7.3), the sequence decreases to $\lambda_{2}$ if $x_{0} \in\left(\lambda_{2}, \infty\right)$ and increases to $\lambda_{2}$ if $x_{0} \in$ $\left[t_{0}, \lambda_{2}\right)$. If we take $x_{0}=2$ and $x_{0}=t_{0}=\frac{2 r}{r+1}$, then the $x_{1}$ are $2-\frac{1}{2^{r}}$ and $2-\frac{1}{2^{r}}\left(1+\frac{1}{r}\right)^{r}$, respectively. Therefore

$$
2-\frac{\left(1+\frac{1}{r}\right)^{r}}{2^{r}}<\lambda_{2}<2-\frac{1}{2^{r}}
$$

By the continuity, if there is another zero of $p(t)$ on or outside of the unit circle, then there must exist a $k \in(0,1]$ such that a zero $t \neq 1$ of $t^{r}(t-2)=$ $-k$ is on the unit circle.

Let $t=\cos \theta+i \sin \theta$. Then

$$
\cos \theta+i \sin \theta=t=2-\frac{k}{t^{r}}=(2-k \cos (r \theta))+i k \sin (r \theta)
$$

and by taking absolute value both sides, we have

$$
1=4-4 k \cos (r \theta)+k^{2} \quad \text { or } \quad k^{2}-4 k \cos (r \theta)+3=0 .
$$

If $\cos (r \theta)=1$ then $k=1$ is a solution and (7.4) gives us $t=1$. So we only need to consider the root $2\left(\cos (r \theta)-\sqrt{\cos ^{2}(r \theta)-(3 / 4)}\right)$ for $\cos (r \theta) \in$ $(\sqrt{3} / 2,1)$. The others are obviously not in $(0,1]$. Let

$$
f(x)=2\left(x-\sqrt{x^{2}-(3 / 4)}\right) .
$$

Since $f(1)=1$ and $f^{\prime}(x)=2\left(1-\frac{x}{\sqrt{x^{2}-(3 / 4)}}\right)<0$ for $x \in(\sqrt{3} / 2,1), f(x)>$ 1 for all $x \in(\sqrt{3} / 2,1)$. Therefore all the zeros of $p(t)$ other than $\lambda_{1}, \lambda_{2}$ are located inside the unit circle.

For each $\lambda_{j}$, let $\xi_{j}=\left(z_{1}, \ldots, z_{r+1}\right)^{T}$ be the eigenvector. Then $A \xi_{j}=\lambda_{j} \xi_{j}$ gives us

$$
z_{2}=\lambda_{j} z_{1}, \quad z_{3}=\lambda_{j} z_{2}, \quad \ldots, \quad z_{r+1}=\lambda_{j} z_{r} .
$$

Let $z_{1}=1$, we then have

$$
\xi_{j}=\left(1, \lambda_{j}, \ldots, \lambda_{j}^{r}\right)^{T} .
$$


Proof of Theorem 3.5. Let $\left(\lambda_{i}, \xi_{i}\right), i=1, \ldots, r+1$, be the eigenpairs of $A$ where $\xi_{j}$ is given by $(3.4)$, and let $b=(0,0, \ldots, 1)^{T}$. Then $b=\sum_{j=1}^{r+1} \alpha_{j} \xi_{j}$ where $\left(\alpha_{1}, \ldots, \alpha_{r+1}\right)^{T}$ is the solution of

$$
\left(\begin{array}{cccc}
1 & 1 & \cdots & 1 \\
\lambda_{1} & \lambda_{2} & \cdots & \lambda_{r+1} \\
\vdots & \vdots & & \vdots \\
\lambda_{1}^{r} & \lambda_{2}^{r} & \cdots & \lambda_{r+1}^{r}
\end{array}\right)\left(\begin{array}{c}
\alpha_{1} \\
\alpha_{2} \\
\vdots \\
\alpha_{r+1}
\end{array}\right)=\left(\begin{array}{c}
0 \\
0 \\
\vdots \\
1
\end{array}\right)
$$

The determinant of the coefficient matrix is the Vandermonde determinant $V\left(\lambda_{1}, \ldots, \lambda_{r+1}\right)$, and if we replace the $j$ th column by $b$ and expand along it, the determinant is $(-1)^{r+1+j} V\left(\lambda_{1}, \ldots, \lambda_{j-1}, \lambda_{j+1}, \ldots, \lambda_{r+1}\right)$. By the Crammer's rule and the formula for Vandermonde determinants,

$$
\begin{aligned}
\alpha_{j} & =(-1)^{r+1+j} \frac{\prod_{1 \leq k<l \leq r+1, k \neq j, l \neq j}\left(\lambda_{l}-\lambda_{k}\right)}{\prod_{1 \leq k<l \leq r+1}\left(\lambda_{l}-\lambda_{k}\right)} \\
& =\frac{(-1)^{r+1+j}}{\prod_{k=1}^{j-1}\left(\lambda_{j}-\lambda_{k}\right) \prod_{k=j+1}^{r+1}\left(\lambda_{k}-\lambda_{j}\right)} \\
& =\frac{1}{\prod_{k \neq j}\left(\lambda_{j}-\lambda_{k}\right)} .
\end{aligned}
$$

Note that

$$
p(t)=t^{r+1}-2 t^{r}+1=\prod_{i=1}^{r+1}\left(t-\lambda_{i}\right), \quad \text { and } \quad p^{\prime}(t)=\sum_{i=1}^{r+1} \prod_{k \neq i}\left(t-\lambda_{k}\right) .
$$

So

$$
\begin{aligned}
\alpha_{j}=\frac{1}{\prod_{k \neq j}\left(\lambda_{j}-\lambda_{k}\right)} & =\frac{1}{p^{\prime}\left(\lambda_{j}\right)} \\
& =\frac{1}{(r+1) \lambda_{j}^{r}-2 r \lambda_{j}^{r-1}}=\frac{1}{\lambda_{j}^{r-1}\left((r+1) \lambda_{j}-2 r\right)}
\end{aligned}
$$

Let $X_{m}=\left(x_{m}, \ldots, x_{m+r}\right)^{T}$. Then the system $(3.1)$ is

$$
X_{m+1}=A X_{m}+b\left(2^{m}\right), \quad N(m+r, r)=b^{T} X_{m}, \quad X_{0}=b .
$$


By the solution of linear discrete systems, we have

$$
\begin{aligned}
X_{m} & =A^{m} X_{0}+\sum_{i=0}^{m-1} A^{i} b\left(2^{m-1-i}\right) \\
& =\sum_{j=1}^{r+1} \alpha_{j} \lambda_{j}^{m} \xi_{j}+\sum_{i=0}^{m-1}\left(\sum_{j=1}^{r+1} \alpha_{j} \lambda_{j}^{i} \xi_{j}\right) 2^{m-1-i} \\
& =\sum_{j=1}^{r+1}\left(\lambda_{j}^{m}+\sum_{i=0}^{m-1} \lambda_{j}^{i} 2^{m-1-i}\right) \alpha_{j} \xi_{j} \\
& =2^{m-1} \sum_{j=1}^{r+1}\left(2\left(\frac{\lambda_{j}}{2}\right)^{m}+\sum_{i=0}^{m-1}\left(\frac{\lambda_{j}}{2}\right)^{i}\right) \alpha_{j} \xi_{j} \\
& =2^{m-1} \sum_{j=1}^{r+1}\left(\left(\frac{\lambda_{j}}{2}\right)^{m}+\sum_{i=0}^{m}\left(\frac{\lambda_{j}}{2}\right)^{i}\right)^{m} \xi_{j} \\
& =2^{m-1} \sum_{j=1}^{r+1}\left(\left(\frac{\lambda_{j}}{2}\right)^{m}+\frac{1-\left(\lambda_{j} / 2\right)^{m+1}}{1-\left(\lambda_{j} / 2\right)}\right) \alpha_{j} \xi_{j} \\
& =2^{m} \sum_{j=1}^{r+1} \frac{1+\left(\lambda_{j} / 2\right)^{m}-2\left(\lambda_{j} / 2\right)^{m+1}}{2-\lambda_{j}} \alpha_{j} \xi_{j} .
\end{aligned}
$$

So

$$
\begin{aligned}
N(n, r) & =b^{T} X_{n-r}=2^{n-r} \sum_{j=1}^{r+1} \frac{1+\left(\lambda_{j} / 2\right)^{n-r}-2\left(\lambda_{j} / 2\right)^{n-r+1}}{2-\lambda_{j}} \alpha_{j} b^{T} \xi_{j} \\
& =2^{n-r} \sum_{j=1}^{r+1} \frac{\lambda_{j}\left(1+\left(\lambda_{j} / 2\right)^{n-r}-2\left(\lambda_{j} / 2\right)^{n-r+1}\right)}{\left(2-\lambda_{j}\right)\left((r+1) \lambda_{j}-2 r\right)}
\end{aligned}
$$

Proof of Theorem 3.6. Let $X_{m}=\left(x_{m+1}, \ldots, x_{m+r}\right)^{T}$. Then the system (3.6) is

$$
X_{m+1}=A X_{m}+b\left(2^{m+1}\right), \quad H(m+r, r)=b^{T} X_{m}, \quad X_{0}=2 b,
$$

where $A$ is the $r \times r$ matrix defined by $(3.3)$ and $b=(0,0, \ldots, 1)^{T}$. Similar to the proof of last theorem, $b=\sum_{j=1}^{r} \alpha_{j} \xi_{j}$ where

$$
\alpha_{j}=\frac{1}{\lambda_{j}^{r-2}\left(r \lambda_{j}-2(r-1)\right)}
$$


and

$$
\begin{aligned}
X_{m} & =A^{m} X_{0}+\sum_{i=0}^{m-1} A^{i} b 2^{m-i} \\
& =2^{m} \sum_{j=1}^{r}\left(2\left(\frac{\lambda_{j}}{2}\right)^{m}+\sum_{i=0}^{m-1}\left(\frac{\lambda_{j}}{2}\right)^{i}\right) \alpha_{j} \xi_{j} \\
& =2^{m+1} \sum_{j=1}^{r} \frac{1+\left(\lambda_{j} / 2\right)^{m}-2\left(\lambda_{j} / 2\right)^{m+1}}{2-\lambda_{j}} \alpha_{j} \xi_{j} .
\end{aligned}
$$

So

$$
\begin{aligned}
H(n, r) & =b^{T} X_{n-r} \\
& =2^{n-r+1} \sum_{j=1}^{r} \frac{1+\left(\lambda_{j} / 2\right)^{n-r}-2\left(\lambda_{j} / 2\right)^{n-r+1}}{2-\lambda_{j}} \alpha_{j} b^{T} \xi_{j} \\
& =2^{n-r+1} \sum_{j=1}^{r} \frac{\lambda_{j}\left(1+\left(\lambda_{j} / 2\right)^{n-r}-2\left(\lambda_{j} / 2\right)^{n-r+1}\right)}{\left(2-\lambda_{j}\right)\left(r \lambda_{j}-2(r-1)\right)} .
\end{aligned}
$$

Proof of Theorem 3.8. Note that $p(t)=0$ is equivalent to $t=1-\frac{1}{2^{r+1} t^{r}}$. Similar to the proof of Theorem 3.4, if we define a sequence

$$
x_{n+1}=1-\frac{1}{2^{r+1} x_{n}^{r}}
$$

then $\left\{x_{n}\right\}$ increases to $t_{1}$ if $x_{0} \in\left[r /(r+1), t_{1}\right)$ and decreases to $t_{1}$ if $x_{0} \in$ $\left(t_{1}, \infty\right)$. By taking $x_{0}=\frac{r}{r+1}$ and $x_{0}=1$, we have

$$
1-\frac{\left(1+\frac{1}{r}\right)^{r}}{2^{r+1}}<t_{1}<1-\frac{1}{2^{r+1}} .
$$

If $r$ is even, then $p\left(\frac{-1}{2^{1+\frac{1}{r}}}\right)=\frac{-1}{2^{(r+1)^{2} / r}}$ and

$$
\begin{aligned}
p\left(-\frac{1}{2^{1+\frac{2}{r}}}\right) & =-\left(\frac{1}{2^{1+\frac{2}{r}}}\right)^{r+1}-\left(\frac{1}{2^{1+\frac{2}{r}}}\right)^{r}+\frac{1}{2^{r+1}} \\
& >-2\left(\frac{1}{2^{1+\frac{2}{r}}}\right)^{r}+\frac{1}{2^{r+1}}=0 .
\end{aligned}
$$

So there is a zero $t_{3} \in\left(\frac{-1}{2^{1+\frac{1}{r}}}, \frac{-1}{2^{1+\frac{2}{r}}}\right)$. 
$p(t)=0$ can also be written as

$$
t^{r+1}=t^{r}-\left(\frac{1}{2}\right)^{r+1}
$$

Consider root locus of

$$
t^{r+1}=k\left(t^{r}-\left(\frac{1}{2}\right)^{r+1}\right)
$$

As $k$ changes from 0 to $\infty, r+1$ zeros of $t^{r+1}$ break away along the directions of $\theta=\frac{(2 k+1) \pi}{r+1}, k=0,1, \ldots, r$. The complex conjugate pair along the directions of $\frac{\pi}{r+1}$ and $\frac{(2 r+1) \pi}{r+1}=2 \pi-\frac{\pi}{r+1}$ meet at some place to the right of $\frac{1}{2^{1+\frac{1}{r}}}$ on the real axis and become two real zeros, one moves toward right to $\infty$, and the other moves toward left to $\frac{1}{2^{1+\frac{1}{r}}}$. All the other $r-1$ zeros move to $\frac{1}{2^{1+\frac{1}{r}}} e^{2 k \pi i}, k=1,2, \ldots, r-1$. By root locus techniques [6], the breakaway point is given by $\frac{d}{d t} \frac{t^{r}-\left(1 / 2^{r+1}\right)}{t^{r+1}}=0$, i.e. $t=\frac{1}{2^{1+\frac{1}{r}}}(1+r)^{1 / r}$, and the corresponding $k=\frac{1}{2^{1+\frac{1}{r}}}\left(1+\frac{1}{r}\right)(1+r)^{1 / r}$, which is less than 1 if $r>1$. So for $r>1$, as $k$ changes from 0 to 1 , a pair complex conjugate zeros meet at $t=\frac{1}{2^{1+\frac{1}{r}}}(1+r)^{1 / r}$, then one moves toward right becomes $t_{1}$, and the other moves toward left and becomes $t_{2}$. Therefore $\frac{1}{2^{1+\frac{1}{r}}}<t_{2}<\frac{1}{2^{1+\frac{1}{r}}}(1+r)^{1 / r}$.

Finally, let $t=\rho e^{i \theta}$ in the equation $2^{-r}=t^{r}-t^{r+1}$ and separate the real and imaginary parts, we have two equations

$$
\begin{aligned}
\rho^{r} \cos (r \theta)-\rho^{r+1} \cos ((r+1) \theta) & =\frac{1}{2^{r}} \\
\rho^{r} \sin (r \theta)-\rho^{r+1} \sin ((r+1) \theta) & =0 .
\end{aligned}
$$

Eq. (7.7) is equivalent to

$$
\rho \sin ((r+1) \theta)=\sin (r \theta)
$$

Substitute it into (7.6) we have

$$
\rho^{r}=\frac{\sin ((r+1) \theta)}{2^{r+1}(\sin (r+1) \theta \cos r \theta-\cos (r+1) \theta \sin r \theta)}=\frac{\sin ((r+1) \theta)}{2^{r+1}(\sin \theta)}
$$

and substitute it back into (7.8) we have

$$
\sin ^{r}(r \theta) \sin \theta-2^{-r-1} \sin ^{r+1}((r+1) \theta)=0 .
$$


Note that for each $k$ that satisfies $1 \leq k<r / 2$, the left-hand side of (7.10) is less than 0 when $\theta=\frac{2 k \pi}{r}$ and is greater than zero when $\theta=\frac{(2 k+1) \pi}{r+1}$. So (7.10) has a solution in each of

$$
\left(\frac{2 k \pi}{r}, \frac{(2 k+1) \pi}{r+1}\right), \quad k \in \mathbb{N} \cap[1, r / 2) .
$$

These and their conjugates are all the complex zeros of $p(t)$.

Let

$$
g(\theta)=\frac{\sin ((r+1) \theta)}{\sin \theta} .
$$

We show $0<g(\theta)<r+1$ for all $\theta \in\left(\frac{2 k \pi}{r}, \frac{(2 k+1) \pi}{r+1}\right)$. Clearly $g(\theta)>0$ on the interval, and $g\left(\frac{2 k \pi}{r}\right)=1, g\left(\frac{(2 k+1) \pi}{r+1}\right)=0$. So we only need to show that $g\left(\theta_{0}\right)<r+1$ at the critical point $\theta_{0}$ of $g$. If $\sin \left((r+1) \theta_{0}\right) \geq(r+1) \sin \theta_{0}$, i.e. if

$$
\frac{\sin \left((r+1) \theta_{0}\right)}{(r+1) \sin \theta_{0}} \geq 1
$$

then

$$
\begin{aligned}
\cos \left((r+1) \theta_{0}\right) & =\sqrt{1-\sin ^{2}\left((r+1) \theta_{0}\right)} \leq \sqrt{1-(r+1)^{2} \sin ^{2} \theta_{0}} \\
& <\sqrt{1-\sin ^{2} \theta_{0}}=\cos \theta_{0}
\end{aligned}
$$

which means that

$$
\frac{\cos \left((r+1) \theta_{0}\right.}{\cos \theta_{0}}<1
$$

However

$$
0=g^{\prime}\left(\theta_{0}\right)=\frac{(r+1) \sin \theta_{0} \cos \left((r+1) \theta_{0}\right)-\sin \left((r+1) \theta_{0}\right) \cos \theta_{0}}{\sin ^{2} \theta_{0}}
$$

which is equivalent to

$$
\frac{\sin \left((r+1) \theta_{0}\right)}{(r+1) \sin \theta_{0}}=\frac{\cos \left((r+1) \theta_{0}\right.}{\cos \theta_{0}},
$$

a contradiction. Therefore we must have $g\left(\theta_{0}\right)<r+1$ and by (7.9),

$$
\rho=\frac{(g(\theta))^{1 / r}}{2^{1+\frac{1}{r}}} \leq \frac{\left(g\left(\theta_{0}\right)\right)^{1 / r}}{2^{1+\frac{1}{r}}}<\frac{(1+r)^{1 / r}}{2^{1+\frac{1}{r}}}
$$

for any solution $\theta$ of (7.10). 


\section{References}

[1] K. Binswanger and P. Embrechts, Longest runs in coin tossing, Insurance Math. Econom. 15 (1994), 139-149.

[2] M. Dehmer and A. Mowshowitz, Bounds on the moduli of polynomial zeros, Applied Mathematics and Computation, Vol. 218 (8), 2011, 41284137.

[3] M. Dehmer, On the location of zeros of complex polynomials, Journal of Inequalities in Pure and Applied Mathematics, Vol. 7 (1) (2006).

[4] P. Erdos and P. Revesz, On the length of the longest head-run, Topics in information theory (Second Colloq., Keszthely, 1975), pp. 219-228. Colloq. Math. Soc. Janos Bolyai, Vol. 16, North-Holland, Amsterdam, 1977.

[5] J. Komlos and G. Tusnady, On sequences of "pure heads", Ann. Probability 3 (1975), 608-617.

[6] B. C. Kuo, Automatic Control Systems, 3rd edition, Prentice-Hall, Inc., 1975.

[7] I. Fazekas, Z. Karacsony, and Z. Libor, Longest runs in coin tossing. Comparison of recursive formulae, asymptotic theorems, computer simulations, Acta Univ. Sapientiae, Mathematica, 2 (2010), 215-228.

[8] W. Feller, An Introduction to Probability Theory and Its Applications, Volume 1, 3rd Edition, Wiley, 1968.

[9] M. F. Schilling, The longest run of heads, College Math. J. 21 (1990), 196-207.

Department of Mathematics and Statistics, Texas Tech University

LubBock, TX 79409, USA

E-mail address: sally.nguyen@ttu.edu

Department of Mathematics and Statistics, Texas Tech University

LUBBOCK, TX 79409, USA

E-mail address: alex.wang@ttu.edu

Department of Mathematics and Statistics, Texas Tech University

LUBBOCK, TX 79409, USA

E-mail address: clyde.f.martin@ttu.edu 